\title{
Sobre: cumplimiento de la quimioprofilaxis antipalúdica en ASPFOR XXXIII
}

\section{Excmo. Sr. Director,}

La Sanidad Militar está experimentando un cambio radical en los últimos años, adaptándose desde su antigua misión, fundamentalmente asistencial, a una Sanidad eminentemente operativa ${ }^{1}$. El nivel político exige, especialmente en las circunstancias actuales, centrar los recursos de las Fuerzas Armadas en lo operativo ${ }^{2}$. Nos alegra profundamente por tanto, observar el interés creciente mostrado en las publicaciones en la revista Sanidad Militar en las enfermedades infecciosas, y particularmente en la Medicina Tropical y del Viajero, de evidente prioridad en la atención a la fuerza desplegada en zona de operaciones (ZO).

Resulta de gran interés a este respecto el informe publicado por los Comandantes Médicos Dña. Carolina Arcos y D. Tomás Salinas "Cumplimiento de la quimioprofilaxis antipalúdica en ASPFOR XXXIII”, publicado en el no 2, volumen 71, año 2015 de la revista Sanidad Militar. Igualmente resulta satisfactorio comprobar como el despliegue de Oficiales Médicos especialistas destinados en Órgano Central en los ROLE1 en ZO, aunque aparta temporalmente a los mismos de las tareas propias de su especialidad complementaria, estimula a compañeros como los Ctes. Arcos y Salinas para retomar y actualizar sus conocimientos específicos logístico-operativos e incluso aportar investigación original al conocimiento científico en las escasas horas libres que una misión tan dura como ISAF permite al personal del Cuerpo Militar de Sanidad. Artículos como éste son a nuestro juicio el principal aliciente que acerca a los compañeros civiles a la lectura de esta revista incrementando aún más su impacto y nivel científico.

Con objeto de aportar nuestro grano de arena a este último punto, no obstante, nos vemos obligados a recordar -en relación con el informe de los Ctes. Arcos y Salinas- nuestra carta publicada el n ${ }^{\circ}$ 4, volumen 69, año 2013 de la revista Sanidad Militar ${ }^{3}$ en respuesta a un estudio original de Rico $\mathrm{P}$ et $a l^{4}$. El paludismo o malaria es efectivamente una enfermedad producida por protozoos del género Plasmodium sp. (falciparum, ovale, vivax y malariae). Y por una quinta especie, Plasmodium knowlesi ${ }^{5-7}$. Dado que nuestra carta previa ${ }^{3}$, hizo un breve repaso del descubrimiento e importancia asistencial y logístico-operativa del conocimiento de dicho patógeno, no nos redundaremos al respecto.

Con espíritu constructivo, e insistiendo en alabar el encomiable e indiscutiblemente útil para la ciencia médica esfuerzo en realizar investigación científica en $\mathrm{ZO}$, echamos también en falta ampliar información metodológica. Nos resultaría de interés conocer el número de personal encuestado, y dado que la información se obtuvo de los cuestionarios de salud de los reconocimientos médicos de fin de misión, nos queda la duda de si se solicitó autorización a los encuestados para extraer datos con fines científicos de dichos cuestionarios.

Volviendo a la cuestión de las cinco especies de Plasmodium patógenas en el hombre, sí que nos gustaría aprovechar para incidir sobre dos cuestiones. La primera, mencionar la actual disyunti- va respecto a si nuestra anterior afirmación podría quedar obsoleta en breve. Recientemente estudios moleculares han llevado a subcatalogar al Plasmodium ovale en dos subespecies, $P$. ovale curtisi y $P$. ovale walkerii ${ }^{8}$, indistinguibles morfológicamente pero que según indicios clínicos retrospectivos podrían tener diferencias en el curso clínico ${ }^{9}$ que hacen que algunos expertos las consideren ya dos especies separadas. Habría en tal caso que corregir la clásica respuesta a la pregunta del número de Plasmodium patógenos para el hombre, que no habrían pasado de cuatro a cinco, sino a seis.

La segunda, recalcar la importancia de la formación continuada y de la necesidad de dedicar personal específico de la Red Hospitalaria Militar a agrupar la patología infecciosa tropical dentro del espectro de la amenaza NBQ y a explorar mecanismos para difundir los conocimientos adquiridos por dicha experiencia clínica al personal facultativo previo a su despliegue en ZO. Nuestras FAS contarán en breve, probablemente a pleno rendimiento cuando esta carta sea publicada, con unas instalaciones para el tratamiento de patología infecciosa del máximo nivel mundial ${ }^{10}$, pero no debemos olvidar que el flujo de pacientes de los ROLE1 a través de la cadena sanitaria de evacuación hasta nuestro ROLE4, el Hospital Central de la Defensa "Gómez Ulla", debe acompañarse de un flujo de conocimiento científico que se dirija en sentido inverso a través de mecanismos como pudieren ser futuras actividades formativas o como sin duda es nuestra revista de Sanidad Militar.

$\mathrm{Y}$ es que en ciencia no hay dogmas, y si los hay casi indefectiblemente el progreso de la ciencia los desmiente. El esfuerzo en el continuo reciclaje es parte del trabajo de cualquier médico, y especialmente del que atiende a una población de riesgo en entornos hostiles y cambiantes de un día a otro según las decisiones políticas y estratégicas. Asistencia, sí... e investigación, y formación continuada. Son y deben ser parte de nuestra instrucción y adiestramiento.

\section{BIBLIOGRAFÍA}

1. Tarilonte E. Médicos y militares. Revista Española de Defensa 2015; 317 30-33

2. Rajoy, M. "Nuestras Fuerzas Armadas y su futuro". ABC 12 de octubre de 2011 Disponible en http://www.abc.es/historico-opinion/index.asp?ff=20111012\&i $\mathrm{dn}=1501930381177$ (visitado el 4 de septiembre de 2015).

3. Membrillo FJ, Fe A, Gámez A, Perea C. Sobre: Protocolo de extracción de ADN en lotes de 10 mosquitos para la identificación de Plasmodium spp. mediante qPCR. Sanid Mil 2013; 69(4): 283-4.

4. Pérez Rico A, Lacasa J, Rubio JM, Ruiz S, Vega J. Protocolo de extracción de ADN en lotes de 10 mosquitos para la identificación de Plasmodium spp. mediante qPCR. Sanid mil 2013; 69(2): 77-81.

5. Knowles R, Das Gupta BM. A study of monkey-malaria and its experimental transmission to man. Ind Med Gaz 1932; 67:301-21.

6. Singh B, Kim Sung L, Matusop A, et al. A large focus of naturally acquired Plasmodium knowlesi infections in human beings. Lancet 2004; 363:1017-24.

7. Cox-Sing J, Davis T, Lee KS, Shamsul S, Matusop A, Ratnam S, Rahman H, Conway DJ, Singh B. Plasmodium knowlesi Malaria in Humans Is Widely Distributed and Potentially Life Threatening. Clin Infect Dis 2008; 46:165-71. 


\section{CARTAS AL DIRECTOR}

8. Sutherland CJ, Tanomsing N, Nolder D, Oguike M, Jennison C, et al. Two nonrecombining sympatric forms of the human malaria parasite Plasmodium ovale occur globally. J Infect Dis 2010; 210(10): 1544-1550.

9. Rojo-Marcos G, Rubio-Munoz JM, Ramirez-Olivencia G, Garcia-Bujalance S, Elcuaz-Romano R, et al. Comparison of imported Plasmodium ovale curtisi and P. ovale wallikeri infections among patients in Spain, 2005-2011. Emerg Infect Dis 2014; 20(3): 409-416.

10. Fe A, Membrillo FJ. Unidad de aislamiento hospitalario de alto nivel. Necesidad y características. Sanid Mil 2014; 71(2): 74-76.
Fco. Javier Membrillo de Novales.

Cap. Médico.

Antonio Fe Marqués. Cor. Médico.

Unidad NRBQ-Infecciosas, Hospital Central de la Defensa. Madrid. España

\section{RÉPLICA DE LOS AUTORES}

Excmo. Sr Director,

Como no puede ser de otra manera, hemos leído con mucho interés y atención la carta enviada a su revista por el Coronel Fe y el Capitán Membrillo.

En relación a sus afirmaciones, comentarios y dudas, queríamos hacer las siguientes consideraciones, siempre, por supuesto, con el mismo ánimo constructivo con el que las han manifestado:

\section{Primera consideración:}

En relación con las especies de Plasmodium entre las que se incluye P. knowlesi y la probable catalogación de P. ovale en P. ovale curtisi y P. ovale walkerii, únicamente queremos mencionar que la fuente de la que nos servimos fue la de la OMS en su actualización de 2014.

Hemos leído, tras conocer estas nuevas categorías de Plasmodium, la carta que publicaron en esta revista el Col. Fe y el Cap. Membrillo en relación con el asunto, reconociendo con ello su importancia para el ámbito asistencial y logístico-operativo presente y futuro.

\section{Segunda consideración:}

Respondiendo al interés metodológico de su carta, queremos hacerles saber que, efectivamente, la información se obtuvo de los cuestionarios de salud de los reconocimientos médicos de fin de misión.

En todo momento se informó a los sujetos entrevistados de que los datos recogidos se utilizarían para el citado informe publicado en esta revista, ascendiendo el número del personal entrevistado por nuestra parte a 427.

\section{Tercera consideración:}

Para los autores, lo más importante y en lo que queríamos poner el foco con este informe, es en la inadecuada quimioprofilaxis antipalúdica que se realizó en esta rotación de ASPFOR XXXIII, así como que no nos parece una situación aislada, sino que se viene dando en Zona de Operaciones ( $\mathrm{ZO}$ ) de una forma frecuente a pesar de la extensa experiencia en misiones internacionales que ya poseemos.

De ahí que concluyamos con la necesidad de un replanteamiento de la situación para conseguir un cumplimiento adecuado de la quimioprofilaxis antipalúdica en $\mathrm{ZO}$.

No queremos dejar de señalar que nos ha resultado gratificante poder constatar el interés creado por nuestro informe, hecho que nos estimula a seguir manteniendo nuestro espíritu crítico pero constructivo en las próximas misiones que seguro vendrán, tratando de aportar, aunque sea mínimamente, al conocimiento científico.

Finalmente, nos complace compartir aquí en su revista, la invitación que nos ha hecho llegar el Comité Internacional de Medicina Militar, ICMM Scientific Council, en sus siglas en inglés, para publicar nuestro artículo en su revista "International Review of the Armed Forces Medical Services".

Carolina Arcos Sánchez Cte Médico. Servicio de Neurología

Francisco Tomás Salinas Vela

Cte Médico. Servicio de Radiodiagnóstico Hospital General de la Defensa “Orad y Gajías”. Zaragoza 